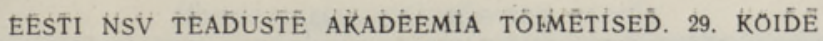
FUOSIKA * MATEMAATIKA. 1980, NR. 1

ИЗВЕСТИЯ АКАДЕМИИ НАУК ЭСТОНСКОН ССР. ТОМ 29 ФНЗИКА * МАТЕМАТИКА, 1980, № 1

\title{
НОРМАЛЬНЫЕ МНОГООБРАЗИЯ АЛГЕБР
}

\author{
(Представлена А. Хумалом)
}

Пусть a - некоторое многообразие $\Omega$-алгебр. Многообразие а назовем нормальным, если множество нульарных операций из $\Omega$ пусто и все тождества, определяющие многообразие a, таковы, что в левую и правую части рассматриваемого тождества входят одни и те же переменные. Алгебра $A$ из а называется проективной $\left[{ }^{1}\right]$ (с. 156), если всякая диаграмма

$$
\begin{gathered}
A \\
P \rightarrow Q^{\pi},
\end{gathered}
$$

состоящая из $\Omega$-алгебр из а и их гомоморфизмов, где $\pi-$ эпиморфизм, продолжается до коммутативной, т. е. существует гомоморфизм $\psi: A \rightarrow P$ такой, что $\pi \psi=\varphi$. Инъективная $\Omega$-алгебра из а определяется двойственным образом.

В настоящей статье рассматриваются вопросы описания нормальных многообразий $\Omega$-алгебр, каждая из которых проективна (инъективна). Аналогичные вопросы решались для многообразий модулей над кольцами $\left[{ }^{2}\right]$ (теорема 4.2), полигонов над моноидами $\left[{ }^{3-5}\right]$, полумодулей над полукольцами $\left[{ }^{6,7}\right]$, минимальных абелевых многообразий $\Omega$-алгебр с нулем $\left[{ }^{8}\right]$, а также для категорий функторов из малой категории в категорию множеств [ $\left.{ }^{9}\right]$.

Оказывается, требование, чтобы все $\Omega$-алгебры из нормального многообразия a были проективными, соответственно инъективными (фактически мы будем это требовать лишь для конечно порожденных алгебр из а), накладывает условие на сигнатуру $\Omega$. А именно, в этом случае система $\Omega$ состоит только из унарных операций. Этот результат позволяет свести поставленный вопрос к соответствующему вопросу для полигонов над моноидами, решеннюму Л. А. Скорняковым [ $\left.{ }^{3}\right]$.

Пусть а - нормальное многообразие $\Omega$-алгебр и $\Omega \neq \varnothing$. Введем одну простую конструкцию, которая понадобится нам в дальнейшем.

Пусть $A$ - произвольная $\Omega$-алгебра из многообразия а и $\Theta$ $(\Theta \notin A)$ - некоторый символ. Рассмотрим множество $A^{*}=A \cup \Theta$ и определим на нем операции из $\Omega$ следующим образом: для произвольной $n$-арной операции $\omega \in \Omega$ и любых $a_{1}, \ldots, a_{n} \in A^{*}$ положим

$a_{1} \ldots a_{n} \omega=\left\{\begin{array}{cc}a_{1} \ldots a_{n} \omega \in A, \text { если } a_{i} \in A \text { для всех } i=1, \ldots, n ; \\ \Theta & \text { в противнөм случае. }\end{array}\right.$ 
Таким образом, формулы (1) задают на $A^{*}$ структуру $\Omega$-алгебры. Легко видеть, что $\Omega$-алгебра $A^{*}$ принадлежит многообразию а. Действительно, всякое тождество, выполняющееся в a, в силу нормальности $\mathfrak{a}$, имеет вид

$$
p\left(x_{1}, \ldots, x_{n}\right)=q\left(x_{1}, \ldots, x_{n}\right),
$$

где $p$ и $q-$ некоторые главные производные операции $\left[{ }^{10}\right]$ (с. 155) от системы операций $\Omega$. Тогда, ввиду (1), для произвольных $a_{1}, \ldots, a_{n} \in$ $\in A^{*}$ в случае, если $a_{i} \in A$ для каждого $i=1, \ldots, n$, будет

$$
p\left(a_{1}, \ldots, a_{n}\right)=q\left(a_{1}, \ldots, a_{n}\right),
$$

поскольку $A \in \mathfrak{a}$, а если $a_{i}=\Theta$ для некоторого $i(1 \leqslant i \leqslant n)$, то $p\left(a_{1}, \ldots, a_{i-1}, \Theta, a_{i+1}, \ldots, a_{n}\right)=\Theta \quad$ и $\quad q\left(a_{1}, \ldots, a_{i-1}, \Theta, a_{i+1}, \ldots\right.$ $\left.\ldots, a_{n}\right)=\Theta$, т. e.

$$
p\left(a_{1}, \ldots, a_{n}\right)=q\left(a_{1}, \ldots, a_{n}\right) .
$$

Следовательно, $A^{*} \in$ a.

Введенную $\Omega$-алгебру $A^{*}$ назовем расширением $\Omega$-алгебры $A$ внешним нулем $\Theta$.

Т е о р е а 1. Если все конечно порожденные $\Omega$-алгебры из нормального многообразия а ингективны, то $\Omega$ состоит только из унарных операций.

Д ок азз а тельст в о. Пусть все $\Omega$-алгебры из а инъективны. Предположим, что существует $n$-арная $(n \geqslant 2)$ операция $\omega \in \Omega$, и покажем, что это приводит к противоречию.

Пусть $A-$ произвольная конечно порожденная $\Omega$-алгебра из а и $A^{*}$ - расширение алгебры $A$ внешним нулем $\Theta$. Через $B=A^{*} \times A^{*}$ обозначим прямой квадрат $\Omega$-алгебры $A^{*}$. Ясно, что $B \in \mathfrak{a}$. Рассмотрим подмножество

$$
C=\left\{(a, \Theta),(\Theta, a) \mid a \in A^{*}\right\}
$$

в $\Omega$-алгебре $B$. Легко видеть, ввиду (1), что $C$ есть подалгебра в $B$ и, значит, $C \in \mathfrak{a}$. Ясно также, что $C$ конечно порождена. Ввиду инъективности $C$, диаграмма

$$
\underset{\mathrm{I} \downarrow}{C \stackrel{\mathrm{l}}{\rightarrow} B} \text {, }
$$

где $\iota$ - естественное вложение, I - тождественный автоморфизм, продолжается до коммутативной, т. е. существует гомоморфизм $\varphi: B \rightarrow C$ такой, что $\varphi \imath=\mathrm{I}$. Тогда

$$
\varphi(c)=\varphi \iota(c)=\mathrm{I}(c)=c
$$

для любого $c \in C$.

Пусть $a_{1}, \ldots, a_{n}$ - произвольные элементы из $A$ и пусть, для определенности,

$$
\varphi\left(a_{1}, a_{1}\right)=(a, \Theta)
$$

для некоторого $a \in A^{*}$. Для операции $\omega \in \Omega$ имеем в $B$ равенство $\left(a_{1}, a_{1}\right)\left(\Theta, a_{2}\right) \ldots\left(\Theta, a_{n}\right) \omega=\left(a_{1} \Theta \ldots \Theta \omega, a_{1} \ldots a_{n} \omega\right)=\left(\Theta, a_{1} \ldots a_{n} \omega\right)$. (Если элемент $\varphi\left(a_{1}, a_{1}\right)$ имеет вид $(\Theta, a)$, то рассматриваем соотношение, аналогичное последнему, но с переставленными координатами 
элементов из $B$ ). Применяя к элементам из этого соотношения гомоморфизм $\varphi$, учитывая (2) и (3), получим

$$
(a, \Theta)\left(\Theta, a_{2}\right) \ldots\left(\Theta, a_{n}\right) \omega=\left(\Theta, a_{1} \ldots a_{n} \omega\right) .
$$

Преобразование левой части равенства (4) дает

$$
(a, \Theta)\left(\Theta, a_{2}\right) \ldots\left(\Theta, a_{n}\right) \omega=(\Theta, \Theta),
$$

т. е. равенство (4) принимает вид

$$
(\Theta, \Theta)=\left(\Theta, a_{1} \ldots a_{n} \omega\right) .
$$

Отсюда следует $a_{1} \ldots a_{n} \omega=\Theta$. Последнее, ввиду предположения $a_{i} \in A \quad(1 \leqslant i \leqslant n)$, означает, что $\Theta \in A$. Полученное противоречие доказывает теорему.

Аналогичный результат имеет место и в «проективном случае».

Т е о р е а 2. Если все конечно порожденные $\Omega$-алгебры из нормального многообразия а проективны, то $\Omega$ состоит только из унарных операций.

Док а зательст в о. Пусть $G$ - свободная в а алгебра, порожденная одним свободным образующим $g \in G$, и $G^{*}-$ ее расширение внешним нулем $\Theta$. Через $F$ обозначим $\Omega$-алгебру, состоящую из трехмерных векторов с элементами из $G^{*}$, на которых операции из $\Omega$ действуют покомпонентно. Ясно, что $F \in \mathfrak{a}$.

Для произвольных $f \in F, i=1,2,3$, через $f(i)$ обозначим $i$-ю координату вектора $f$. Через $\vec{a}_{i} \in F$ обозначим вектор, на $i$-м месте которого стоит элемент $a \in G^{*}$, а на остальных местах $-\Theta$. Через $B \subset F$ обозначим подалгебру, порожденную элементами $\left\{\bar{g}_{i}, i=1,2,3\right\}$.

Пусть $V$ - свободная $\Omega$-алгебра из $а$, порожденная тремя свободными образующими $v_{1}, v_{2}, v_{3}$. Ввиду проективности $\Omega$-алгебры $B$, диаграмму

$$
\begin{aligned}
& B \\
& \stackrel{\downarrow}{\downarrow} \stackrel{\mathrm{I}}{B},
\end{aligned}
$$

где I - тождественный автоморфизм, $\pi$ - эпиморфизм такой, что $\pi\left(v_{i}\right)=\bar{g}_{i}$, можно продолжить до коммутативной некоторым гомоморфизмом $\varphi: B \rightarrow V$, т. е. $\pi \varphi=\mathrm{I}$. Покажем, что $\varphi\left(\bar{g}_{i}\right)=v_{i}$ для любого $i=1,2,3$. Для этого, ввиду $\pi \varphi\left(\bar{g}_{i}\right)=\mathrm{I}\left(\bar{g}_{i}\right)=\bar{g}_{i}=\pi\left(v_{i}\right)$, нам достаточно показать, что из $\pi(u)=\bar{g}_{i}$ для некоторого $u \in V$ следует $u=v_{i}$.

Действительно, возьмем для определенности $i=1$ и пусть $\pi(u)=\bar{g}_{1}$, $u=p\left(v_{1}, v_{2}, v_{3}\right)$, где $p=p(x, y, z)$ - некоторая главная производная операция от $\Omega$. Покажем, что слово $p$ не содержит букв $y$ и $z$. Действительно,

$$
\bar{g}_{1}=\pi(u)=\pi\left(p\left(v_{1}, v_{2}, v_{3}\right)\right)=p\left(\pi\left(v_{1}\right), \pi\left(v_{2}\right), \pi\left(v_{3}\right)\right)=p\left(\bar{g}_{1}, \bar{g}_{2}, \bar{g}_{3}\right),
$$

т. е. $\bar{g}_{1}=p\left(\bar{g}_{1}, \bar{g}_{2}, \bar{g}_{3}\right)$. Ввиду покоординатного применения операции $p$ к элементам из $B$, для первой координаты вектора $\bar{g}_{1}$ имеем

$$
g=p\left(\bar{g}_{1}(1), \bar{g}_{2}(1), \bar{g}_{3}(1)\right)=p(g, \Theta, \Theta) .
$$

Из свойств элемента $\Theta$ и того факта, что $g \neq \Theta$, следует, что слово $p$ не содержит букв $y$ и $z$, т. е. $p(x, y, z)=p(x)$. Кроме того, из (5) получаем

$$
g=p(g)
$$


Так как $g-$ свободный образующий $\Omega$-алгебры $G$, то равенство (6) есть тождество, выполняющееся в а. Следовательно, $u=p\left(v_{1}\right)=v_{1}$. Тем самым доказано, что $\varphi\left(g_{i}\right)=v_{i}$ для любого $i=1,2,3$.

Предположим теперь, что существует хотя бы одна $n$-арная $(n \geqslant 2)$ операция $\omega \in \Omega$, и покажем, что это приводит к противоречию.

Действительно, легко видеть, что в $B$ выполняются равенства

$$
\bar{g}_{1} \underbrace{\bar{g}_{2} \ldots \bar{g}_{2} \omega}_{n-1}=\bar{\Theta}, \quad \bar{g}_{3} \underbrace{\bar{g}_{2} \ldots \bar{g}_{2}}_{n-1} \omega=\bar{\Theta},
$$

где $\bar{\Theta}$ - вектор, на каждом месте которого стоит символ $\Theta$. Таким образом, в $B$ выполняется равенство

$$
\bar{g}_{1} \bar{g}_{2} \ldots \bar{g}_{2} \omega=\bar{g}_{3} \bar{g}_{2} \ldots \bar{g}_{2} \omega .
$$

Применяя гомоморфизм $\varphi$ к обеим частям этого равенства и учитывая, что $\varphi\left(\overline{\mathrm{g}}_{i}\right)=v_{i}$ для любого $i=1,2,3$, получим

$$
v_{1} v_{2} \ldots v_{2} \omega=v_{3} v_{2} \ldots v_{2} \omega .
$$

Так как $\left\{v_{1}, v_{2}, v_{3}\right\}-$ система свободных образующих $\Omega$-алгебры $V$, то последнее равенство есть тождество в $\mathfrak{a}$, что противоречит предположению о том, что а - нормальное многообразие. Теорема доказана.

Теоремы 1 и 2 показывают, что нормальные многообразия $\Omega$-алгебр, каждая из которых инъективна (проективна), исчерпываются соответствующими многообразиями унарных алгебр. Легко видеть, что совокупность унарных операций образует полугруппу $S$ относительно операции суперпозиции. Две унарные операции из $S$, действующие на алгебрах из а одинаково, будем отождествлять. Другими словами, если в а выполняется тождество $x v=x \mu(v, \mu \in S)$, то будем считать, что $v=\mu$.

Если полугруппа $S$ содержит тождественную унарную операцию $\varepsilon$, то, очевидно, $S$ - моноид. Действительно, $x(\varepsilon v)=(x \varepsilon) v=x v$, $x(v \varepsilon)=(x v) \varepsilon=x v$, следовательно, $\varepsilon v=v \varepsilon=v$. Если $S$ не содержит тождественной операции $\varepsilon$, то добавим ее к $S$ и потребуем, чтобы в а выполнялось тождество $x \varepsilon=x$. Новую полугруппу $S \cup \varepsilon$ снова обозначим через $S$. Теперь каждая алгебра из $а$, очевидно, становится правым полигоном $\left[{ }^{3}\right]$ над моноидом $S$.

Таким образом, описание нормальных многообразий $\Omega$-алгебр, каждая из которых проективна (инъективна), сводится к описанию многообразий правых $S$-полигонов над моноидом $S$, а фактически к описанию моноида $S$, над которым все правые полигоны проективны (инъективны). Такое описание получено в [ $\left.{ }^{3}\right]$, а также может быть получено, как частный случай, из результатов $\left[{ }^{9}\right]$ (см. также $\left.\left[{ }^{4,5}\right]\right)$.

В частности, из теоремы $3\left[{ }^{3}\right]$ вытекает

С л е д с в и е. В нормальном многообразии а все (конечно порожденные) $\Omega$-алгебры проективны тогда и только тогда, когда а есть многообразие множеств и $\Omega$ либо пусто, либо состоит из одной тождественной унарной операции.

«Инъективный случай» формулируется более громоздко.

\section{Л ИТЕРАТ У РА}

1. Кон П., Универсальная алгебра, М., «Мир», 1968.

2. К а р та н А., Э йл е н е р г С., Гомологическая алгебра, М, Изд-во иностр. лит., 1960. 
3. С ко р н я ко в Л. А., Сиб. матем. ж., № 9, $1139-1143$ (1969)

4. F o u n t a i n, I. B., Proc. London Math. Soc., 3, № 28, 28-44 (1974).

5. I s b e 11, J. R., J. Algebra, 23, 228-238 (1972).

6. Ф л я й ш р В. Г., Уч. зап. Тартуск. ун-та, № 366, 42-75 (1975).

7. Фля йш е p В. Г., В кн.: Второй всесоюз. симп. по теории колец, алгебр и модулей, Кишинев, «Штиинца», 1974, с. 62.

8. Fle is her, V., In: Colloquia Mathematica Societatis János Bolyai (17. Contributions to universal algebra), Szeged (Hungary), 1975, p. 113-132.

9. По л и н С. В., Матем. сб., 93, 381-404 (1974).

10. К у р о А. Г., Лекции по общей алгебре, М., «Наука», 1973.

\section{Тартуский государственный университет}

Поступила в редакцию 10/VII 1979

\section{FLJAISER}

\section{NORMAALSED ALGEBRATE MUUTKONNAD}

$\Omega$-algebrate mutkonda a nimetatakse normaalseks, kui $\Omega$ ei sisalda nullaarseid operatsioone ja selle mutkonna iga defineeriva samasuse vasak pool sisaldab samu mutujaid kui parem pool. Artiklis on näidatud, et kui normaalse $\Omega$-algebrate muutkonna kõik löplikult moodustatud algebrad on projektiivsed (injektiivsed), siis $\Omega$ koosneb ainult unaarsetest operatsioonidest. Selliste unaarsete algebrate muutkondade kirjeldus vastavate monoidide abil on teada $\left[{ }^{3}\right]$.

\section{FLJAISER}

\section{NORMAL VARIETIES OF ALGEBRAS}

A variety $\boldsymbol{a}$ of $\Omega$-algebras is regarded as normal if the set of all nullary operations from $\Omega$ is empty and for every identity in $A$ the sets of variables in its both sides coincide. It is shown that if in a normal variety of $\Omega$-algebras all finitely generated algebras are injective (projective) then $\Omega$ consists of only unary operations. The description of such varieties of unary algebras in terms of properties of the monoid formed by the unary operations from $\Omega$ is known $\left[{ }^{3}\right]$. 